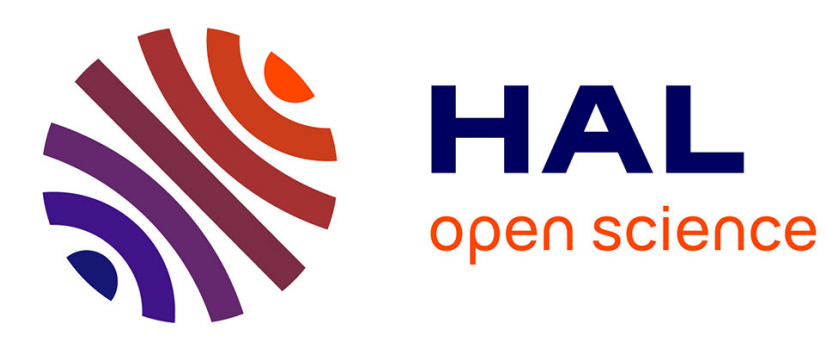

\title{
From Digital camera to Computational Photography
}

Henri Maître

\section{To cite this version:}

Henri Maître. From Digital camera to Computational Photography. SPIE Eighth International Symposium on Multispectral Image Processing and Pattern Recognition, Oct 2013, Wuhan, China. pp.891701-14. hal-01103749

\section{HAL Id: hal-01103749 https://hal-imt.archives-ouvertes.fr/hal-01103749}

Submitted on 15 Jan 2015

HAL is a multi-disciplinary open access archive for the deposit and dissemination of scientific research documents, whether they are published or not. The documents may come from teaching and research institutions in France or abroad, or from public or private research centers.
L'archive ouverte pluridisciplinaire HAL, est destinée au dépôt et à la diffusion de documents scientifiques de niveau recherche, publiés ou non, émanant des établissements d'enseignement et de recherche français ou étrangers, des laboratoires publics ou privés. 


\title{
From Digital camera to Computational Photography*
}

\author{
Henri Maître \\ Institut Mines-Télécom - Télécom-ParisTech - CNRS LTCI \\ 46 rue Barrault - 75013 Paris - France
}

9 septembre 2013

\begin{abstract}
Summary : Digital cameras are now more than 30 years old. They benefit from an exceptional popularity that the conventional film cameras never obtained. Digital cameras differ from conventional analog cameras with respect to several distinctive features, some of which stand as undeniable improvements, some remaining on the contrary as brakes towards their use in every technical field. However, in the last years, these distinctive features are fostering new techniques of world observation, these techniques relying merely on the openings proposed by the new sensors. Here is the domain of Computational Photography. Before we present a critical survey of the objectives of computational photography, a review of the distinctive features of digital cameras is made, in order to determine where progress may be expected.
\end{abstract}

Key-words : Digital camera, image processing, computational photography, computational imaging

*Presented at SPIE Eighth International Symposium on Multispectral Image Processing and Pattern Recognition 26 -27 October 2013 Wuhan, China. 


\section{Definitions}

We denote as "Analog camera" the conventional cameras which make use of films or plates. The emblematic prototype of analog camera is the traditional " $24 \times 36 \mathrm{~mm}$ " reflex camera, but the complete range of devices is covered by these word, from the cheapest disposable device to the large format professional view-camera $(4 \times 4 \mathrm{~cm}, 6 \times 6 \mathrm{~cm}$, and more $\ldots)$

We denote as "Digital camera" all the portable image acquisition systems which make use of a solid sensor (CCD or CMOS). The digital camera family thus also covers very different devices ranging from mobile phones, compacts, bridges, hybrids, reflex to professional equipments.

We denote by "digital image processing" 1 the enhancement and transformation methods which, starting from an image provide another image of similar nature, but where some components are enhanced. Such are the methods for contrast or resolution enhancement,for chromatic correction or selection, for frequential filtering, edge or region detection, pattern recognition, etc.

In contrast with image processing, computational photography or computational imaging $[16,41]$ has for goal to provide, from a digital camera, unusual pieces of information, unusual, i.e. different from those which are given by the camera, or in a range different from the one we are familiar with or outside the normal sensor range. In this framework, CAT tomography as used in the medical domain, SAR imaging in radar, and many other techniques which are popular in Astronomy, Geophysics or Non Destructive Evaluation (NDE) imaging may be seen as computational photography. However, as opposed to these last methods, computational photography is aiming at using non-professional devices in order to keep close from the user and to make the so-gained progress banal for everybody. Its ultimate goal is indeed to inject the modified software into the user's personal camera.

As we may measure, the frontiers between image processing and computational photography are not only thin, but also fuzzy. There is no good image processing software which do not take into account a careful knowledge on the sensor and the camera parameters. And there will not be technical solutions to computational photography without pieces of software directly imported from the most conventional image processing textbooks.

\section{Digital vs. Analog Cameras}

The supremacy of analog photo on the iconic reproduction of the universe stood for 150 years at least. During this period cameras reached an exceptional development and an unanimously recognized quality whatever the criteria, being scientific (resolution, sensitivity, accuracy, fidelity), technical (robustness, ergonomy, flexibility, interoperability), or even artistic or societal.

Without surprise, we observe that the digital cameras architecture is directly derived today from the analog camera architecture. There is no gap between analog and digital in this domain as it may exist in other close technological domains like telephony for instance. Even more, an attempt has been made to always refer to the analog ancestor when introducing new products in order to exploit the reach heritage of the old technology.

However, when entering into the technological viscera of the available digital camera, we may notice that, even if they are presented alike the old ones, all and every functionality of the digital cameras have been deeply modified. These modifications open doors to deep evolutions of the user's practice. Notice however that some of the emerging opportunities will not be compatible and it is unlikely that the monstrous chimera which could benefit from all these progresses will ever emerge (neither will it probably be desirable that it be looked for).

In broad outline, any camera is made of generic components which are always present (figure 1) :

1. Unless necessary, we will soon abandon the word "digital" since most of the proposed processing are today performed on a computer 


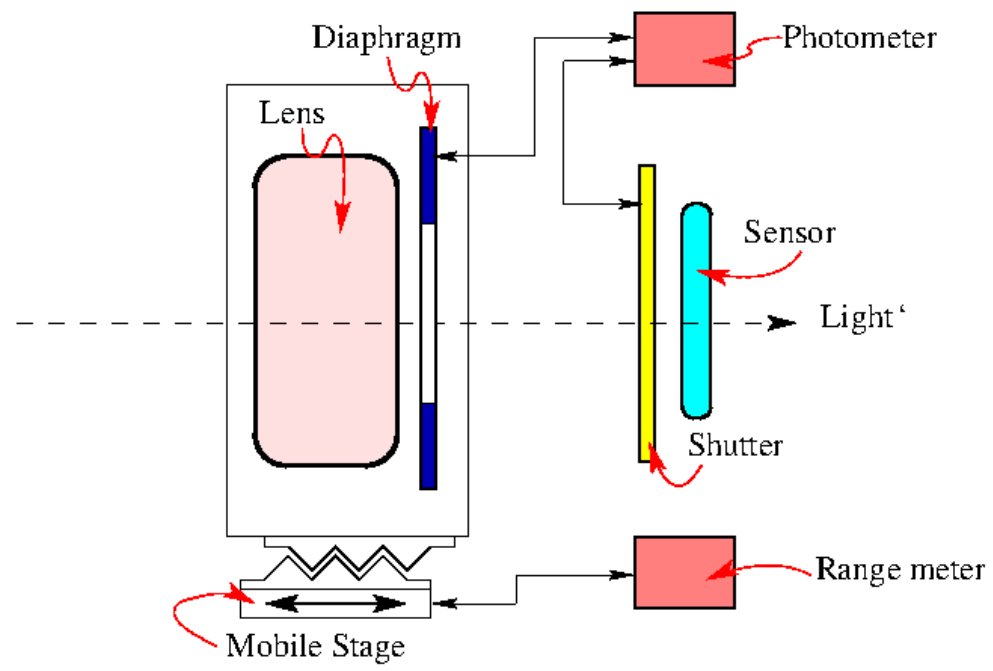

Figure 1 - Block diagram of a camera either analog (the sensor is a film) or digital (the sensor is solid-state).

- a lens or a set of lenses in charge of doing the reduced image of the world,

- a sensor (film, plate or solide-state),

- a shutter to control the sensor lighting,

- a range meter which provides the focusing distance,

- a mobile stage moving the lens or some of the lenses to focus the image according to the range finder instructions,

- a photometer to inform about the light balance in the scene,

- a shutter driven by the photometer, to control the received light.

Additional furniture may be associated with the camera (figure 2) which are useful for the sequel of this presentation :

- optical filters (infra-red and ultra-violet), anti-aliasing filters, located close from the sensor on the optical way;

- a flash lamp to provide an additional and controlled light on the scene;

- associated processing (either photographic (in the dark room) or digital (available either in the camera or off-line (РнотоShop, OpticsPro DxO, ...).

Let us have now a fast overview of the most interesting evolutions of these functions.

\section{$2.1 \quad$ Lens}

Apparently it is a domain where evolutions do not appear clearly. Photographic industry, reduced to a small number of manufacturers, made its best to maintain the market value of a very rich and complete product range designed for analog cameras. Therefore it maintained a reasonable compatibility with digital cameras.

A broad range of lenses (either with fixed focal length or zooms) designed for $24 \times 36$ cameras have been perpetuated even if they were not so well adapted to the smaller sensors available at that time. The emerging "full-field sensors" (i.e. also $24 \times 36$ ), validated this commercial strategy. Notice however that for the many smaller sensors, adapted lenses allow to improve in optical quality (focusing, aberrations, aperture) at comparable price.

We will come back later on the consequences on the image quality of small sensors, but we may right now underline how they interact with lenses. A smaller field of detection for a similar image demands a shorter focal length, and, as a consequence, a lighter and less cumbersome lens (practical consequence), but also, because of technical arguments, as larger openings of the aperture are easier to produce, more luminous lenses, larger depth of focus, and therefore more tolerant and easier focusing. 


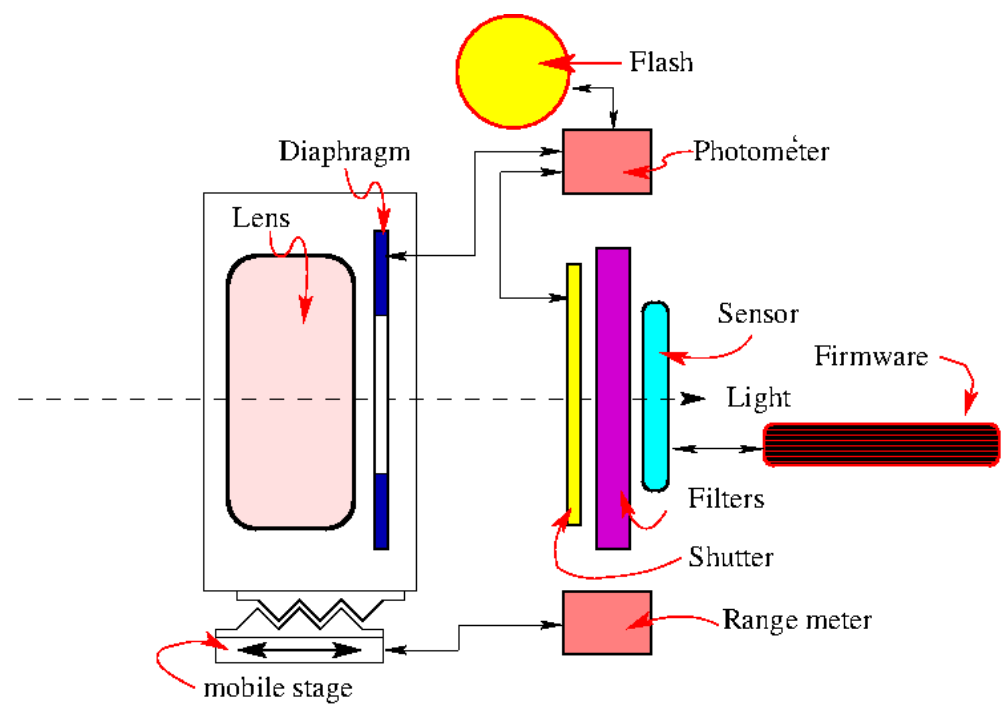

Figure 2 - Additional components associated with the camera : optical filters, additional light source, image processing software of firmware.

Following this track to its ultimate point, simplest cameras (as for instance many phones, tablets and smart phones) use fix lens without focusing, by exploiting the very weak value of the hyper-focal distance $^{2}$. Such a possibility (which was yet exploited with analog cameras, as for instance in the popular Brownie Kodak), makes the diagram of figure 1 much simpler.

Other evolutions also make the most of non-reflex set-up specificity. It is the case of additional lenses which are used "after" the main lens (i.e. in between the lens and the sensor). Although additional lenses introduced "before" the main lens to extend the focal length of the camera are well known from the photographers (they are called teleconverters), these lenses have the opposite role of shortening the focal lens. This benefit is made possible only since non-reflex cameras are mirrorless and allow for shorter focusing than reflex which cannot reduce their flange. Moreover as these cameras are often used with oversized lenses with respect to the sensor size, they allow to shorten the focal without vignetting the image. All together, such lenses are often seen as if they increase the lens aperture (which is wrong), because aperture and focal length are linked by $N=f / D .^{3}$. These additional lenses, by focusing the image on a smaller number of detectors allow to speed up the snapshot.

However, the most important evolution in the domain of photographic lenses is probably to be expected in the domain of deformable lenses. It relies on another paradigm : the variation of the focal length is due to the curvature variation of the lens (according to the equation $1 / f=(n-1)\left(1 / R+1 / R^{\prime}\right.$, $R$ and $R^{\prime}$ being the lens curvatures and $n$ its index). Such lenses are made of liquid or viscous materials and are deformed by electrostatic or magnetic constraints. This way, mobile parts are eliminated, making the system simpler and possibly faster.

\subsection{The photo sensor}

It is rightly seen as one of the key elements in the digital camera, and certainly it experienced the greatest evolution in the recent years. Moreover, nothing let imagine that the speed of its evolution

2. hyper-focal $f_{h}$ is the shortest distance which ensures a focusing until infinite. When a camera is fixed at hyperfocal distance, it provides a focused images from $f_{h} / 2$ to infinite. For a camera with $f$ as focal length and $N$ as numerical aperture, the hyper-focal is : $f_{h} \approx \frac{2 f^{2}}{\varepsilon N}$ where $\varepsilon$ is the tolerance diameter accepted for focusing.

3. They are often sold under commercial names which refer to this possibility to overtake the physical limitations of the lens : "Turbo" or "Booster". See for instance http ://www.dpreview.com/articles/2667195592/first-impressionsmetabones-speed-booster. 
will be reduced in the coming years.

The most important achievements of the sensor in the recent years have been the increase of :

- the overall sensor size which will directly be related to the field of view of the camera,

- its resolution, roughly expressed as the distance between adjacent sites on the device,

- the total number of pixels which is roughly the product of the two previous figures,

- the signal dynamics,

- the signal noise ratio which is somehow related to the camera ISO sensitivity,

- the shooting rate.

Sensor size and resolution Briefly speaking, sensors come with a great diversity in size : from some few millimeters for instance in many phones, up to several centimeters for the largest (see figure 3), they now cover the whole range of photographic films, up to the medium-format. However, sensors above the "full size" $(24 \times 36 \mathrm{~mm})$ remain very expensive and are dedicated to professional use. While the sensor dimensions increased, their resolution decreased. Therefore the pixel number follows a steep growth. In 2013, below 5 Mpixels, the sensors can only be found in cheap telephones; usual cameras are around 15 Mpixels and most of the "good" cameras are above 20 Mpixels.

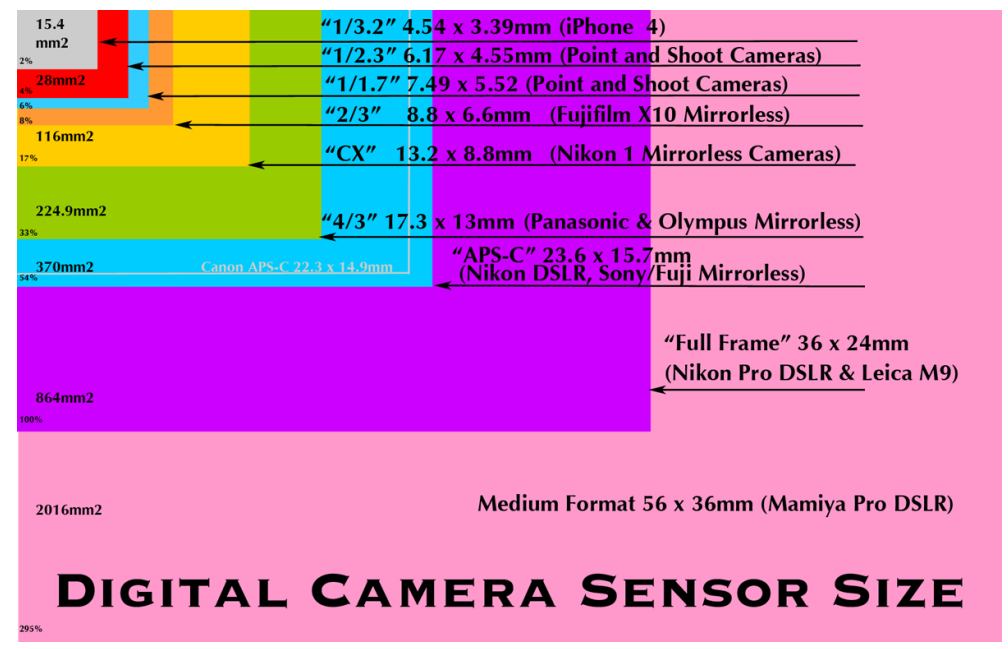

Figure 3 - From $15 \mathrm{~mm}^{2}$ to $2000 \mathrm{~mm}^{2}$ ranges the surface of the most common cameras as in 2011 (from Matt Sassamatt - 2011).

Signal Noise Ratio Required by the miniaturization of the photo-sites which receive some rare photons only, the signal dynamics was also the object of a great attention : with better semiconductors, with better converters, with high protection against parasites, with excellent signal processing algorithms. Sensitivities over 10000 ISO allows for rater good images, and some cameras offer to go above 100000 ISO. Just to remember, films barely venture above 1600 ISO.

As a consequence of the increase in signal dynamics and quality, the quantization on 8 bit per pixel as required by most of the standards (compression, transmission, display), appears today too narrow and many cameras allow for the acquisition with 10, 12 or 14 bits (in RAW mode).

Photo detector The use of CMOS sensors instead of the more traditional CCD, fostered architecture modifications, with processors distributed at line or row heads, therefore paralleling signal collection and conversion. Higher image rates resulted, allowing for instance HD video rates for videos or rafale mode up to 10 images per second in full frame still images. Notice that, if hesitations welcomed the arrival of CMOS as image sensors in the cameras, instead of CCD, they are now favored by 
cameras companies because of their flexibility to participate to complex architectures. Against odds, questions may be ask today on the future of CCD.

Color Acquisition This domain appears as commercially stabilized, but remains scientifically open. The choice of a spatial matricing of color channels has been almost everywhere adopted, and Bayer matrix (which distributes 2 sites for Green, 1 for Blue and 1 for Red on any $2 \times 2$ sensor sub-matrix) is obviously adopted as the standard. However, alternative solutions exist which suggest to adopt a full color non-multiplexed sensor (FOVEON/SIGMA sensor or "True Color" project from NiKON). They exhibit many advantages that expert photographers do appreciate. Less drastically different, alternative chromatic matrices (figures 4 and 5) present also noticeable qualities, either by proposing different masks (in Kodak experimental sensors, the Green is replaced by a Panchromatic sensor) or by adding primaries (in SONY DsCF628 sensor, a fourth color (Emerald) is associated to Red, Green and Blue).
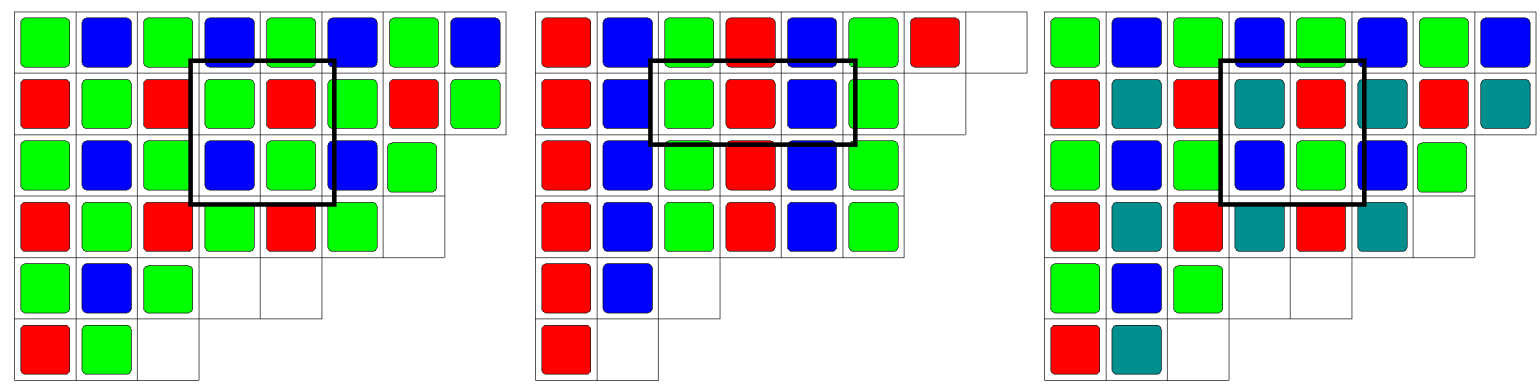

Figure 4 - From left to right : Bayer's matrix, stripped matrix, Sony DSCF628 with 4 colors (one over 2 Green sites is replaced by an Emerald site.) For each matrix, the black box represents the minimal periodic pattern.
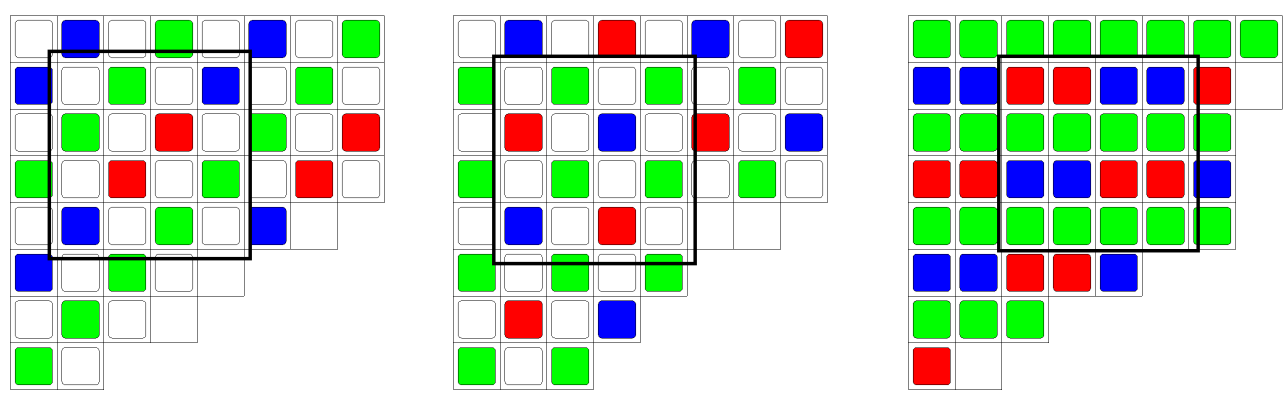

Figure 5 - From left to right : 2 proposals from Kodak, with panchromatic sites (white), ESR matrix from Fuji, which may be reconfigured in 3 different modes by grouping the sites.

\subsection{Shutter}

The shutter use to be a mechanical device. It is now often electronic which provides important insights to future applications. An electronic command open doors for an individual per-pixel command (a complex operation however). It allows also for very flexible opening commands, shorter apertures and precise connections with flash. Associated with mechanical shutters, electronic shutter will probably be root to many coming innovations.

\subsection{Diaphragm}

Up to now, the diaphragm has not been the object of modifications in the commercial cameras. We will see however that it is the place of many attempts to improve the camera performances [31]. 


\subsection{Range measuring}

Many different solutions exist today : image superposition, contrast analysis, phase measurement of time of flight. Exploiting the digital signal (either directly on the image sensor or on an additional device) provided facilities for new and powerful range finders with complex strategies around a given target or within the whole image. Simultaneous or alternate use of different modes (either active or passive) allows to overcome difficult situations with low light, uniform or periodical surfaces, reflecting or transparent materials. Many cameras base their focusing after having collected more than 100 measurements and having identified the context (landscape or portrait, sport, close range photo, etc.)

\subsection{Photometer}

By using solutions similar to the ones used for the range measurement (several digital sensors distributed over the image field), the photometer provides different solutions (however, more global decision). The photometer has to face another difficult problem : the decision on the color balance which is often obtained by determining the nature of the dominant illuminant.

\section{$2.7 \quad$ Filters}

In the previous Sections, we associated arbitrarily chromatic filters with sensors. However other filters are needed in a digital camera, to reduce the light band-pass to the visible domain and avoid that the sensor be dazzled by unwanted radiations. The hot filter prevents against infra-Red while the cold filter cuts the ultraviolet wavelengths (figure 6).

Less essential are the anti-aliasing psf the point spread function over several adjacent photo-sites to avoid a too broad image band-pass with respect to Shannon-Nyquist requirements.
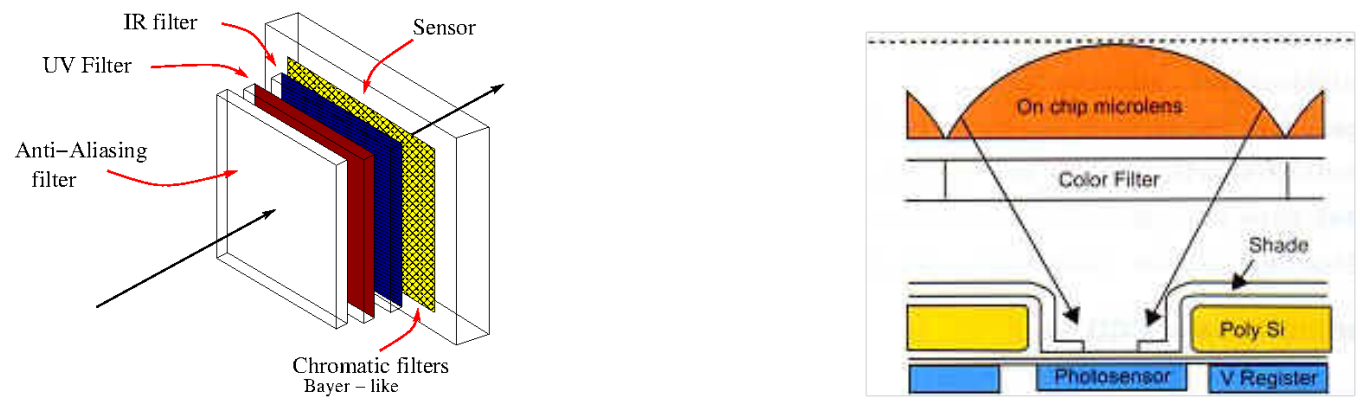

Figure 6 - Optical filters ahead of the sensor : hot filter (IR), cold filter (UV), anti-aliasing filter (on left). The chromatic matrix is made of an array of filters and micro-lenses to increase the efficiency of the measure (on right).

\subsection{Software and Firmware}

Their is a huge demand for computation inside the camera. A CPU is in charge of the the user interface (GUI) (menus, graphics, displays) ; it allows to navigate in the previously recorded images. It is in charge of the image acquisition functions, of its compression (Ziv-Lampel, JPEG, MPEG4). It takes decisions about aperture, pose, white balance, focusing, stabilization of the image. It performs a first level of image processing : correction of aberrations, vignetting, colorimetry. It may perform tracking, face recognition ; it determines the most likely context : indoor/outdoor, landscape/portrait, 
sport, etc. and new functionalities appear on every new model ${ }^{4}$.

The embedded power is mostly distributed on multicore processors and the pressure of new functionality (GPS, gyro-meters, internet connections) is urging for more and more power.

New modern cameras, as every digital devices, require regular uploading of software, not only for its CPU, but also for its many drivers (lens, flash, meters). It may appear strange that a lens needs an updated release, but its focusing strategy, or its stabilization algorithms should follow the evolution of the camera software. Upgrading is made by the manufacturer, using internet connections. Therefore a camera may be seen as a device with a possible access for expert hobbyists as well as professional companies. They propose alternative software (for instance demosaicking) to the manufacturer's one or original functionality (like color handling, solarisation, etc.) which may be plugged in your camera.

As a consequence (and as a good introduction to next Section!) some proposal have been made to fully open a camera so that the user - not necessarily an expert - has an access to most of the functionality ${ }^{5}$.

\section{Computational Photography}

Resolution and pixel number improvement, increase of sensitivity, flexible range measurement, accurate incoming-light measurement, flexibility in the image analysis, centralized management of aperture, of zooming, of stabilization, integration of preprocessing, user-friendly interface, easy connection with the digital world, all this accounts in the assessment of digital photography. It is not few, and it has been enough to gain the largest part of the photo market (but not all!), to invite itself in products where it was not expected (computers, phones, tablets), to drastically modify professional and industrial technological equipments, even if they were only loosely connected to the world of photography. It is indisputably a major technical evolution which has driven major economic and societal evolutions. Under these circumstances, why the emergence of a computational photography which seems to come quite late and which will unlikely be very beneficial to the success of the digital photo market? Besides the marketing interest of the developed techniques for the market renewal, the answer may be to be looked for out of the conventional photo market, in the scientific domain and in the technical instrumentation domain.

The computational photo emergence is the result of the assessment that these new functionality exceptionally exploited by the photographic community, can indeed do more than just "nice pictures". It is possible to exploit them further by diverting a little bit these components and devices to address new measures which will provide new pieces of information which will open doors to new progresses not only in the photo domain, but also in other not well identified domains. Computational Photography is a scientific cascaded betting and it not clear that it will be successful. We will examine in the next lines some facets of the computational photo objectives.

\subsection{Full light field}

A great attention has been paid these last years on two problems, closely linked but different :

- how to obtain a focusing within the whole image, whatever the position of the objects participating to the scene (the so-called plenoptic image),

- how to simultaneously determine the photometric level of a pixel and its 3D position (the so called 3D imagery).

As expressed by its name : Full Light Field the objective is to measure by photo the whole observed volume in the same that holography makes it with coherent optics. This objective has a

4. As in 2013 : smile detection, simultaneous detection and tracking of several faces, and even ... dogs and cats detection, etc.

5. see for instance the Franken camera : http ://graphics.stanford.edu/papers/fcam/. 
large panel of potential applications, and it is not surprising that industrial products (RAYTRIX [39], LYTRO [18]) or prototypes (ADOBE, MERL or CAFIDIS cameras) yet exist.

Among the proposed solutions for these two problems, the measure of an image stack, each image taken with a limited depth of focus, with focal range varying from the closest to the furthest point of the scene, emerged as a possible intermediate objective (this project was addressed for instance in $[24,38])$. Image stacks may be obtained from different means :

- following the pioneering idea of G. Lippman [33] in 1908, by use of micro-lens matrices, as for instance in [29],

- by translating the sensor along the optical axis in the image space [32], or, symmetrically moving the lens [36],

- using coded apertures on the lens (instead of a circular-shaped diaphragm, allowing a selection of rays in a given direction with respect to the optical axis [31, 32, 40],

- determining the focusing blur, a classical subject [37], revisited with new tools in the recent years $[14,27]$,

- using spherical aberrations of the lenses [43], or exploiting a chromatic coding by different focusing of the R, G, B channels [5, 22, 45],

- by combining several of these methods.

Associated demands linked to the image stacks are of different origin. As said before, the availability of an in-all-point focused image as well as the disponibility of the distance-to-camera information are the highest demands. Other needs concern the reconstruction of an image plane at a given focal length not in the stack, the tracking in 3D space of a specific object, the reconstruction of on-focus images on non-planar surfaces, etc.

\subsection{High Dynamic Range Images (HDR)}

For many applications the observed scenes exhibit very high dynamics between high lights and shadows. A challenge is to acquire, transmit and reproduce such dynamics to the user. Human visual system is remarkably adapted to follow the high light excursions, but as long as the information is transmitted 8bit/sample quantization as proposed to day by most of the cameras, and seen as standard for transmission are far insufficient for this purpose. Two main streams of questions are addressed today : how to measure HDR and how to visualize it while maintaining dynamics (the so called tone mapping).

Many studies, reported in [2], aimed at tackling the task by expanding or reconstructing the sensor response either by using separate channels for high and low keys, or by temporally multiplexing signals after prespecified or random shutter sequences, or coded apertures, or by spatially multiplexing the signals (as in the Fuji ESR camera), etc. [4, 21, 26]. It should be noted that, new sensor architectures (Kodak or Fuji, figure 5) have been explicitly developed to address the HDR problem in case of difficult illumination situation.

\subsection{Extended resolution}

It was said that the sensor resolution improved a lot in recent years, however, the demand for larger pixel numbers is still important and not only for commercial reasons. Progress is looked for in several directions : demosaicking remains a field where improvement may be reached, restoration outside the sampling rate frequency is also expected, based on a better knowledge (sub pixel) of the impulse response and the noise, at last proposal are made to better exploit the optical path and its relation to aliasing.

Demosaicking Theoretical aspects of matrix acquisition are drastically limiting the use of conventional tools of Shannon-Nyquist theory to digital cameras. Without a strong optical filtering (very low aperture) (left figure 7), aliasing is mandatory with any matrix, and even more when color-multiplexed 
like in Bayer masking (especially in the Red and Blue channels (figure 7 on right)) .
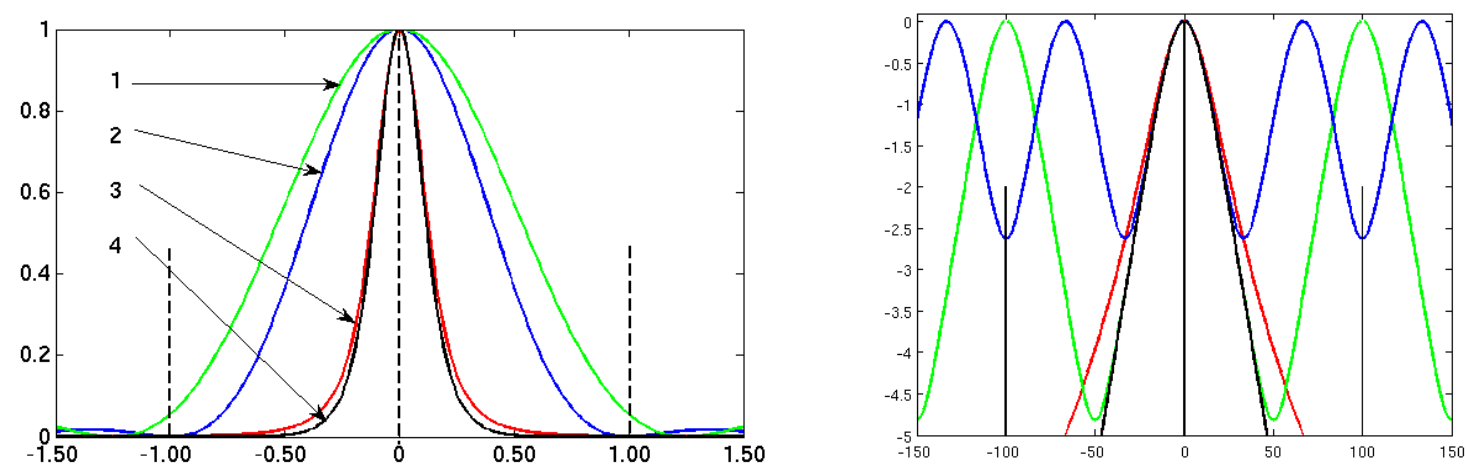

Figure 7 - Power density spectrum of an image as a function of frequencies. On the left : before periodisation by photo-site matrix : image signal spectrum (1) is maximal at 0 frequency, (2) after a filtering by the diaphragm, and (3) after integration on the photo-site cells (a 3/4 ratio was chosen between the integration cell and the photo-site step). As a convention, the frequency introduced by the sensor period is for $u \in[-1,1]$. After the two filtering the signal is described by curve (4).

On the right : after matrix integration due to photo-site repetition (and in logarithmic scale), the signal is now periodized. Red and black curves : as in the left. Green : the signal if sampled with a Bayer filter (Red and Blue channels $=1 / 2$ band aliasing), and with a strip matrix (2/3 aliasing).

A great attention has been paid to the demosaicking problem. The camera manufacturers propose (patented) algorithms of excellent quality and highly efficient for real time processing. They make the best of the signal content and of the knowledge they have on the signal acquisition (psf, noise, ...). They heuristically exploit the complementarity and correlations between channels. Recent proposals (see figure 8) have been made for heavier off-line processing which may benefit from larger contextual decisions to guarantee the continuity of patterns without destroying fine details and randomness of textures $[7,19]$.

Super-resolution Super-resolution is the ultimate goal of many techniques dedicated to improving the quality of digital images. As the sensors do not verify Nyquist sampling conditions, signal outside the sampling band remains in the acquired image which informs about very high frequencies. Super-resolution which aims at exploiting these residual pieces of information, is really effective only in favorable situations as for instance when a diversity of image sources is available. It may be the case with color images when the three channels are quite redundant and exhibit different phases; and the demosaicking algorithms are indeed performing an exact super-resolution. It may also be the case when multiple expositions have been captured, either by moving the target or the sensor. Such situations (which also encompass the full-light-field case), proposed interesting tracks for super-resolution. Besides theoretical works based on compressed sensing and sparse representations [8], interesting practical solutions have been proposed, based on random distribution of sensors or randomly shaped sensors to guarantee a kind of orthogonality between measures [42].

Impulse Response and Noise Important fundamental studies are devoted to precisely characterizing sensor noise to make filtering algorithms more efficient. Excellent algorithms (often patented by manufacturers) are now available, which are directly beneficial to the high ISO sensors previously presented. Noise identification [3, 34], as well as sub-pixel psf determination [14] are necessary steps for improving the filtering. Dedicated targets for the measurement of the psf are necessary to guarantee the optimality in existing works [14, 25] (see figure 9). The user will probably have to get ride of the target in a coming future, as suggested in some studies [13].

Another track would be to better conceive the anti-aliasing optical filters (see Section 2.7). Anti 

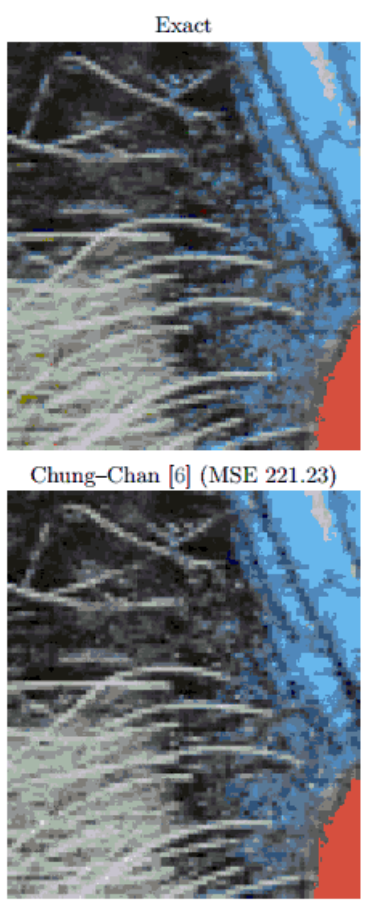

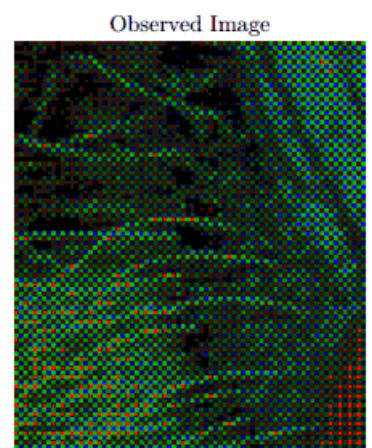

SSD [11] (MSE 220.94)

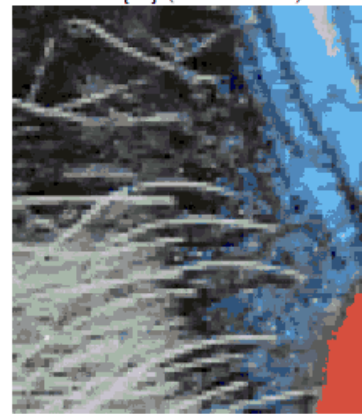

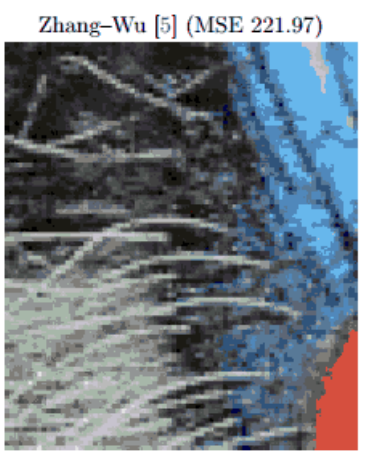

Contour Stencils (MSE 209.95)

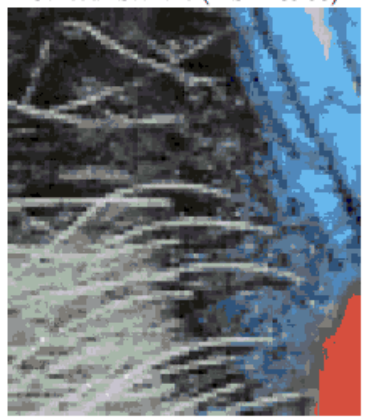

Figure 8 - Demosaicking - from left to right :top - original image, simulated Bayerized image, reconstruction with Zhang and Wu method [46]. Bellow: Chung and Chan method [12], Buadès method [7], Getreuer method [19]. [from IPOL library [19]]
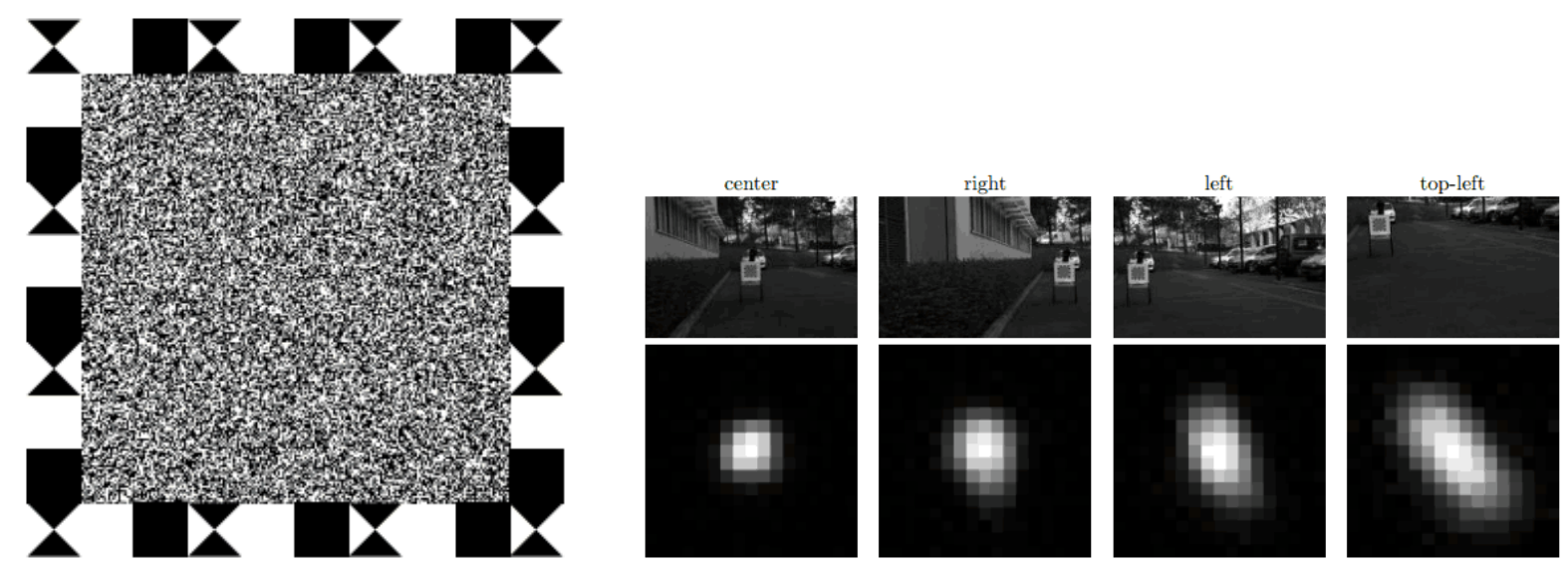

Figure 9 - Resolution target (on the left) and some instances of sub-pixel psf measurement for different distances (on the right) from [14].

aliasing filters are using the birefringent properties of some optical materials (as Quartz or Lithium Niobate) to degrade the original psf by duplicating it (see figure 10). With one properly designed slab, the psf is cast on 2 sites instead of a single one. With two crossed slabs, 4 dots result along the 2 repetition directions of the matrix. Several proposal have been made, either to modify or to suppress them. A main drawback of anti-aliasing filters is that they are physically mounted in front of the sensor and cannot be switched off if necessary. 

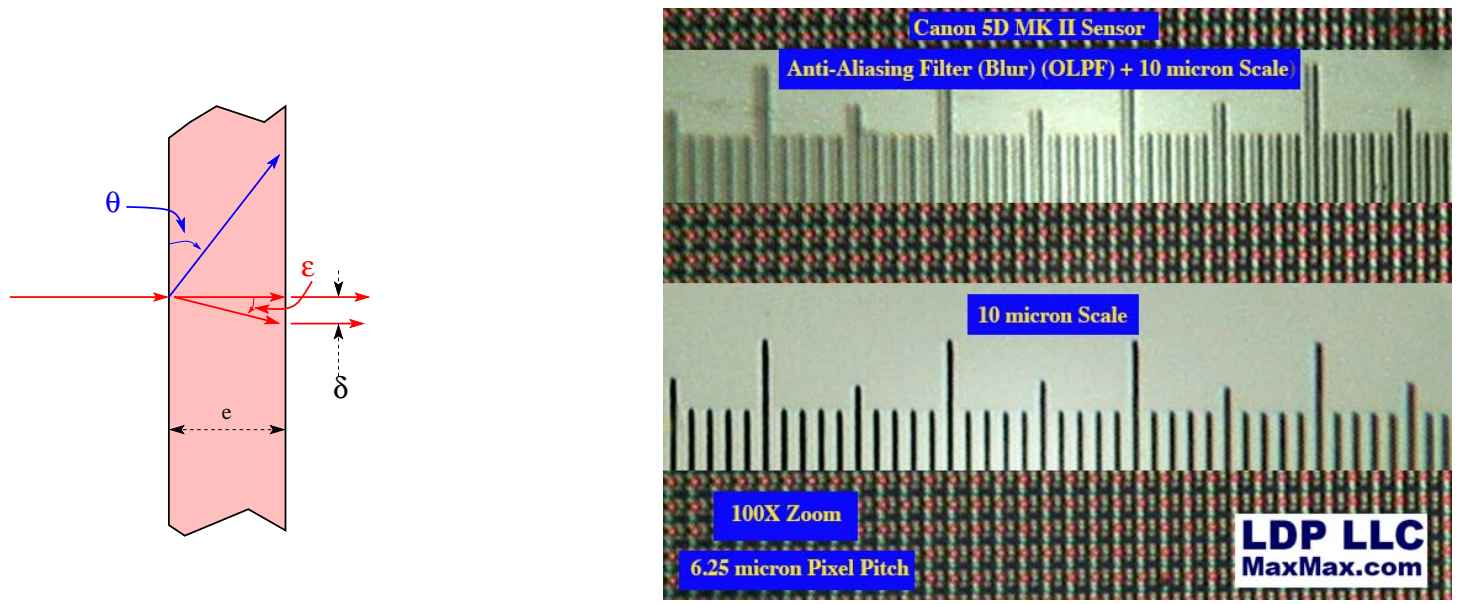

Figure 10 - Anti-aliasing Filter : left, when light goes though the LiNb slab, an ordinary and an extraordinary beam result, with fixed translation $\delta$ in space. On right : images with and without anti-aliasing filters. Above : with the anti-aliasing filter: the image is repeated with a step just equal to the photo-site distance. Below, without anti aliasing filter, the image is much crisper (courtesy MAXMAX : http ://www.maxmax.com/).

\subsection{Color}

It may well be the weakest point of the image acquisition line today and unfortunately it doesn't receive the attention from the scientific community it deserves. We said that the available software on the market are pretty good in the most conventional situations (and sometimes even excellent) for most of the consumer's needs. However, weak points still exist as for instance the reproduction of rare colors (deep blue, indigo, purple, violet, ...), automatic detection of the source content, or the automatic white-balancing. Some applications remain therefore limited, as for instance the reproduction of high quality on printing media, the modification of illuminant, the determination of the intrinsic colorimetric effect of a material, as well as some graphic animations where a given object is moving in front of different light sources. Some works however address the lighting characterization $[17,20,35]$. We may also expect from some novel sensors, as with panchromatic-sites by Kodak, or with 4-primaries by Sony (figures 4 and 5), novel orientations for this domain. Recurs to hyperspectral techniques $[28,10,11]$ which in the colorimetric domain are similar to full light field solution for the focusing problem, is the ultimate track, but requires a very heavy technical investment.

\subsection{Movement management : the "Flutter Shutter"}

Dealing with moving objects is the oldest difficulty with photography. The solution is to be found by shortening the exposure time so that to "freeze" the moving object in a chosen position. This is done at the cost of the light balance, a practical limit to its use, unless additional sources of light (flash lamps) are used. The flash duration in this case fixes the object movement. Interesting effects have been obtained by simultaneously using brief flashes and long mechanical exposures, by choosing the proper time position of the flash during the exposure and eventually to use several delayed flashes. Thanks to the flexibility of the actual electronic shutter control of the modern cameras, these experiments should certainly be expanded with success to address more complex situations where the the successive image trajectories of could be better managed.

Introducing smart stabilization in the camera (either at the lens or at the sensor level) is another way to improve noticeably the impact of vibrations and unwanted movements. Coupled with techniques of target tracking available in some cameras (for the sake of focusing ) or with block matching methods needed for video compression the shortening of exposure time less necessary.

Studies made on flutter shutters [1,23] or those dealing with movement invariant photo- 
graphy [30] are more or less on this track. The most advanced works are using an exposure function which is not a zero/one rectangle function, but another one, which guarantees an easy and regular inversion in a computational restoration. Promising results have been obtained, but with with some paradox consequences [44]. Exploiting the possibility of driving the sensor pixel-per-pixel is another track of great potential. However, it needs rather heavy technological investments.

Developed for providing information about the movement in the scene, flutter shutters also found applications in various fields : field interleaving for video obtained with different sensors, stereo vision or image compression.

\subsection{Compressed Sensing}

In background of many studies devoted to computational photography, lies a major concern of image processing community : the finding of sparse representations well adapted to describe the image. Such representations are actively looked for to gain in compression rates above those (although excellent) of standards (JPEG2000, MPEG4) or to lead to novel acquisition and transmission paradigms, where the compression stage is far simpler (at the possible cost of a heavier decompression stage). Powerful modeling facilities are also expected, dragging along more sophisticated symbolic descriptions, eagerly demanded for efficient indexing, archiving and mining.

All of the previously referenced approaches make an intensive use of multiple and redundant representations far from efficient according to the sparsity criteria. Expecting to reach a representation with a small and sufficient number of signal atoms to reconstruct the broad diversity of observed scenes is present to many of these approaches. Immersing the image in a higher dimension space (plenoptic image or full-light-field or hyper-spectral, could allow to discover more fundamental intrinsic representations as suggested by the compressed sensing wording $[9,15,6])$.

\section{Conclusion}

Only a brief survey of the recent developments to push back the present sensor limits has been made here when a very abundant literature exists. Many applications are fostering such theoretical and experimental developments. Computer vision is the first customer of these results, but video broadcasting and multimedia as well as computer graphics are most interested too. As was noticed in this presentation, the technical tools which help in building computational photography are those which can be found in most image processing text-books. Therefore there is no major difference between image processing and computational photography, at most a more aggressive questioning of the acquisition process along with the acquisition device, a systematic recourse to rather heavy computation to reach the result. Under this lighting, computational photography is probably not sentenced to become a novel scientific field per se. However it appears today as a quite dynamical field of research with innovating actors who deliberately push back the frontiers of their investigation field.

Acknowledgments : Discussions with Maria Cecilia Aguerrebere, Andrès Almansa, Julie DeLON, Yann Gousseau and Saïd LADJAL have been very beneficial to this work.

\section{Références}

[1] A. Agrawal and R. Raskar. Resolving objects at higher resolution from a single motion-blurred image. In IEEE CVPR'O\%, pages 1-8, 2007.

[2] C. Aguerrebere, J. Delon, Y. Gousseau, and P. Musé. Best algorithms for HDR image generation. A study of performance bounds. HAL-00733853-v2, 2012. 
[3] C. Aguerrebere, J. Delon, Y. Gousseau, and P. Musé. Study of the digital camera acquisition process and statistical modeling of the sensor raw data. HAL-00733538-v3, 2012.

[4] C. Aguerrebere, J. Delon, Y. Gousseau, and P. Musé. Simultaneous HDR image reconstruction and denoising for dynamic scene analysis. In IEEE Int. Conference on Computational Photography, ICCP, 2013.

[5] Y. Bando, B.Y. Chen, and T. Nishita. Extracting depth and matte using a color filtered aperture. In ACM SIGGRAPH Asia 2008, page 134, 2008.

[6] C.S. Baraniuk. Compressive sensing. IEEE Signal Processing Magazine, pages 118-121, July 2007.

[7] A. Buades, B. Coll, J.M. Morel, and Sbert C. Self-similarity driven color demosaicking. IEEE trans on PAMI, 18 (6) :1192-1202, 2009.

[8] E.J. Candès and C. Fernandez-Granda. Towards a mathematical theory of super-resolution. Pure \& Applied Mathematics, doi 10.1002/cpa21455, 2013.

[9] E.J. Candès and M.B. Wakin. An introduction to compressive sampling. IEEE Signal Processing Magazine, 25 (2) :21-30, 2008.

[10] C.I. Chang. Hyperspectral imaging : techniques for spectral detection and classification. Springer, 2003.

[11] C.I. Chang. Hyperspectral data exploitation : theory \& Applications. Wiley, 2007.

[12] K.H. Chung and Y.H. Chan. Color demosaicking using variance of color differences. IEEE trans on Image Processing, 15 (10) :2944-2955, 2006.

[13] M. Delbracio, A.Almansa, P. P. Musé, and J.M.Morel. Subpixel point spread function estimation from two photographs at different distances. SIAM Journal on Imaging Science, 5 (4) :1234-1260, november 2012.

[14] M. Delbracio-Bentancor. Two problems of digital image formation. PhD thesis, Ecole Normale Supérieure de Cachan, France, 2013.

[15] D.L. Donoho. Compressed sensing. IEEE trans on Information Theory, 52 (4) :1289-1306, 2006.

[16] F. Durand and R. Szeliski. Guest editors' introduction : Computational photography. IEEE Computer Graphics and Applications, 27 (2), 2007.

[17] M. Ebner. Color Constency. Wiley, 2007.

[18] T. Georgiev, A. Lumsdaine, and S. Goma. Lytro camera technology : theory, algorithms, performance analysis. In Multimedia content and mobile devices, SPIE 8667, 2013.

[19] P. Getreuer. Image demosaicking with contour stencils. IPOL Library : www.ipol.im/pub/art/2012/g-dwcs/, March 2012.

[20] A. Gijsenij, T. Gevers, and J. van de Weijer. Computational color constancy : Survey and experiments. IEEE trans on Image Processing, 20 (9) :2475 - 2489, 2011.

[21] M. Granados, M. Adjin, C. Theobalt, H. Seidel, and H. Lensch. Optimal HDR reconstuction with linear digital cameras. In Computer Vision and Pattern Recognition CVPR, 2010.

[22] F. Guichard, H.-P. Nguyen, R. Tessières, M. Pyanet, I. Tarchouna, and F. Cao. Extended depthof-field using sharpness transport across color channels. In B. G. Rodricks and S. E. Susstrunk (Eds.), editors, IST / SPIE Electronic Imaging (p. 72500N-72500N-12), 2009.

[23] J Holloway, A. Sankaranarayanan, A. Verraraghavan, and S. Tambe. Flutter shutter video camera for compressive sensing of videos. In IEEE int. Conference on Computational Photography, ICCP, 2012.

[24] A. Isaksen, L. McMillan, and S.J. Gortler. Dynamically reparametrized light fields. In SIGGRAPH 2000, pages 297-306, 2000.

[25] N. Joshi, R. Szeliski, and D. Kriegman. PSF estimation using sharp edge prediction. In IEEE Conf. on Comp. Vision $\&$ Pattern Recognition, 2008.

[26] J. Kronander, S. Gustavson, G. Bonnet, and J. Unger. Unified HDR reconstruction from raw CFA data. In IEEE int. Conference on Computational Photography, ICCP, 2013. 
[27] S. Ladjal. Flou et quantification dans les images numériques. PhD thesis, Télécom ParisTech (France), 2005.

[28] D. Landgrebe. Hyperspectral image data analysis. IEEE Signal Processing Magazine, 19 (1) :17$28,2002$.

[29] A. Levin, S.W. Hasinoff, Durand F. Green, P., and W.T. Freeman. 4D frequency analysis of computational cameras for depth of field extension. In ACM SIGGRAPH, 2009.

[30] A. Levin, P. Sand, T.S. Cho, F. Durand, and W.T. Freeman. Motion invariant photography. ACM trans on Graphics, $27: 71,2008$.

[31] C.-K. Liang, T.-H. Lin, B.-Y. Wong, C. Liu, and H.H. Chen. Programmable aperture photography : Multiplexed light field acquisition. ACM Transactions on Graphics, proc. of Siggraph, 27 (3) : $: 55,2008$.

[32] X. Lin, J.-L. Suo, Wetzstein G., Dai Q., and R. Raskar. Coded focal stack photography. In IEEE int. Conference on Computational Photography, ICCP, 2013.

[33] G. Lippman. La photographie intégrale. Comptes Rendus de l'Académie des Sciences, 146 :446$551,1908$.

[34] C. Liu, W.T Freeman, Szeliski R., and S.B. Kang. Noise estimation with single image. In IEEE CVPR'06, pages 901-908, 2006.

[35] B. Mazin, J. Delon, and Y. Gousseau. Illuminant estimation from projections on the Planckian locus. In $C P C V$ workshop, ECCV 2012, 2012.

[36] D. Miau, O. Cossairt, and S. K. Nayar. Focal sweep Videography with deformable optics. In IEEE int. Conference on Computational Photography, ICCP, 2013.

[37] S. K. Nayar and Y. Nakagawa. Shape from focus. IEEE trans on PAMI, 16 (8) :824-831, 1994.

[38] R. Ng. Fourier slice photography. ACM Transactions on Graphics, Proc. of ACM SIGGRAPH, 24 (3) :735-744, 2005.

[39] C. Perwass and L. Wietzke. Single lens 3Dcamera with extended depth of field. In Human Vision and Electronic Imaging, SPIE 8291, 2012.

[40] T. Portz, L. Zhang, and H. Jiang. Random coded sampling for high speed HDR video. In IEEE int. Conference on Computational Photography, ICCP, 2013.

[41] R. Raskar and J. Tumblin. Computational Photography : Mastering New Techniques for Lenses, Lighting, and Sensors. A. K. Peters, Ltd. Natick, MA, USA, 2009.

[42] T. Sasao, S. Hiura, and K. Sato. Super-resolution with random shaped pixels and sparse regularization. In IEEE int. Conference on Computational Photography, ICCP 2013, 2013.

[43] H. Tang and K. Kutulakos. What does an aberrated photo tell us about the lens and the scene? In IEEE Int.Conf. on Computational Photography, ICCP, 2013.

[44] Y Tendero, J.M. Morel, and B. Rougé. The flutter shutter paradox. SIAM Journal Imaging Science, 6 (2) :813-845, 2013.

[45] P. Trouvé. Conception conjointe optique/traitement pour un imageur compact à capacité 3D. $\mathrm{PhD}$ thesis, Ecole Centrale de Nantes (France), 2012.

[46] L. Zhang and X. Wu. Color demosaicking via directional linear m.s.e. estimation. IEEE trans on Image Processing, 14 (12) :2167-2178, 2005. 